\title{
Panel discussion section I
}

\author{
CHAIR: F. Kupka
}

SECTION ORGANIZER \& KEY-NOTE SPEAKER: J.D. Landstreet

Invited SPEAKERs: O.Kochukhov, E.Paunzen, H. Shibahashi

Contribution speakers: S. Vauclair, S. Théado,

\section{Discussion}

SHIBAHASHI: I would like to know how many unknowns - how many equations - you must solve for in the inversion procedure in your new magnetic Doppler imaging method?

Kochukhov: The Doppler imaging problem is intrinsically ill-conditioned. It is not correct to characterize this problem by the number of unknowns. We are using regularization, and regularization effectively introduces a set of additional equations which smoothes the map in our case; in the case of the maximum entropy method, it selects an image with maximum entropy. So actually if I count equations, there are more equations than unknowns.

SHIBAHASHI: What is the resolution of the maps?

Kochukhov: Using Doppler imaging, at a $v \sin i$ of about $30 \mathrm{~km} / \mathrm{s}$, we can resolve about 60 or 80 elements at the rotational equator. Of course, this question must be asked in relation to the observational data that is used for the inversion. For example, for the mapping of pulsations, I used 2000 spectra, so I think I actually had a sufficient number of observational constraints.

NoELS: I would like to ask Shibahashi how sensitive are his results to the choice of the polytrope of $n=3.00$ for the inversion method? If you had chosen another polytrope for the inversion technique, how sensitive are the results to the choice?

Shibahashi: The inversion method which I adopted is linear inversion, so if I took, say, $\mathrm{n}=2.9$, the difference between my inversion and one with $\mathrm{n}=2.9$ is just proportional to the difference between the polytropic indices.

NoEls: I wonder if anyone knows of a simulation in which one takes the helioseismic data for the Sun and artificially limits it to what could be observed in another star? Has anyone taken solar data, say limited to $l=5$ and lower frequencies, to see if one could derive the depth of the convection zone? What precision would we get?

ShibAhAshi: In the case of the Sun, I do not find any big difference if we take the data obtained ten or twenty years ago and compare the results with modern inversions, because the number of eigenmodes detected even at that time was quite large.

In my inversion, using a polytrope, my first intention was to demonstrate the power of asteroseismology, by using quite a lot of eigenmodes, in my case 200. So I intended to reduce the eigenmodes gradually. If I reduce to say $l=2$, the result is quite hopeless. 
NoELs: If you forget all about the Sun, and limit yourself to $1=2$, what would be the precision of the convective envelope?

Shibahashi: The outer part of the convective envelope is quite hard to resolve, but the depth of the convection zone is quite easy.

NoEls: So there is hope for asteroseismology!

MATHYS: I have a question for Kochukhov regarding the modelling of $\alpha$ And? I wonder how you deal with the isotopic structure of mercury? We know that in HgMn stars we may have widely different isotopic compositions from star to star. I think that we could even suspect that we have widely different isotopic compositions on different locations on the surface of $\alpha$ And. The isotopic splitting is large enough that even with rotation you should see some effect on the line profile.

KochuKHOv: First, the effect of isotopic splitting for a $v \sin i$ of $52 \mathrm{~km} / \mathrm{s}$ is really small. I did try to investigate the effect of varying isotopic composition of $\mathrm{Hg}$ over the surface of $\alpha$ And. Admittedly, I used a Q-parameter model, so a simple parameterization of isotopic mixture, and what I find is that the range of $\mathrm{Q}$ is small, from zero to say 0.5. There seems to be no evidence of very large variation of isotopic composition over the surface. In principle, Doppler imaging allows one to reconstruct each isotope individually, but I would not believe these maps. So I restricted myself to Q-parameter, and other maps were reconstructed suing a solar isotopic mixture.

BALOnA: This is a question directed at observers of magnetic Ap stars. According to Shibahashi, the temperature should be cooler around the poles of such a star, and therefore you would expect to see from photometric observations differences in temperature as the star rotates. Is this actually observed?

LANDSTREET: What is observed is that there are photometric variations observed in most Ap stars, of typically 0.02 or $0.03 \mathrm{mag}$, and also spectrum variations. It is not yet clear whether the photometric variations are due exclusively to the spectrum variations, or whether there is some component due to temperature variations. The fact is that we can model spectra of more or less normal stars, such as Sirius, very well, and so we can have real confidence in our models of such stars. In contrast, we cannot yet model spectra of magnetic stars at all satisfactorily, beyond modelling a single line at a time, or perhaps a multiplet. There are so many effects to take into account, such as horizontal inhomogeneities, vertical stratification, and a possibly complex field geometry, that our models match observations rather poorly. Thus I think it is still rather uncertain whether the observed variations have a temperature variation component in them or not.

KochukHOv: I would like to make the following comment. As I understand, in Shibahashi's model, the central issue is suppression of convection at the magnetic poles. It is important to remember that convection becomes quite inefficient when we go above 8000 $\mathrm{K}$, and spots are observed in much hotter stars, so there seem to be two different regions in the HR diagram with very similar phenomenological effects. Concerning the possibility of observing temperature variations, I think this can be done in cool Ap stars by studying variations of Balmer lines as a function of rotation phase, because in cool Ap's the Balmer lines are sensitive to temperature. We should see some systematic variations of Balmer lines if there are cool spots. 
LANDSTREeT: Remember that the Balmer lines may also be influenced by magnetic pressure effects, or by a gradient of mean molecular weight, for example produced by a vertical helium abundance gradient.

KocHUKHOV: If you are thinking about magnetic pressure effects due to the Lorentz force, this effect is limited to hotter Ap stars. The effect is not significant in cool Ap stars simply because the plasma is not ionized sufficiently. We actually calculated model atmospheres of $\mathrm{A}$ and $\mathrm{B}$ stars including the effect of the Lorentz force. We find that for temperatures of about $8000 \mathrm{~K}$ and less there is very little effect of the Lorentz force on atmospheric structure. Effects become significant only at, say, about $11000 \mathrm{~K}$.

LANDSTREET: I think that this is actually fairly uncertain because the origin of the currents is uncertain. There are several possible sources of currents. Unless you know the entire field configuration and how it is changing, it is difficult to be sure that you have accounted for all the currents. You can set lower limits to the currents from particular sources, but I think it is difficult to be sure you know the currents exactly.

KochukHov: The effect does not depend on the currents, it depends on the conductivity.

Landstreet: No, the effect depends on the currents. The Lorentz force is simply $\mathbf{j} \times \mathbf{B}$.

Kochukhov: Let's discuss this later.

TRAMPEDACH: I have a question for Shibahashi about inversions. The density kernels you were using were also negative. Have you played around with other kernels? I believe that it is possible to construct kernels that are almost positive definite, giving a more deterministic inversion.

SHIBAHASHI: What I showed in my talk is a kernel of a certain eigenmode. In the procedure of the inversion we actually make a delta-like function by combining a certain set of eigenmodes. In that sense, the combined density kernel to detect the density profile at a certain layer is positive mainly at that layer, and, ideally, close to zero elsewhere. But actually the kernels of each eigenmode are oscillating between positive and negative for the density kernel, and only positive or zero in the case of the sound velocity kernels.

Adelman: Coming back to the question of surface temperature variations in Ap stars. Some years ago, Leckrone took OAO-2 UV photometry and optical photometry of several magnetic CP stars and showed that integrated flux from those stars was constant to pretty good precision, implying that the effective temperature was not varying by more than some degrees (see Molnar 1973, ApJ 179, 527). I later showed that the error bars for one of the stars could be reduced further if the 5200 A feature, which Leckrone had ignored, was included.

I have a question. What percentage of the metal-poor A stars are considered to be $\lambda$ Boo stars?

PAUnZEN: If you look at Pop I stars, there is some kind of transition between metalpoor stars and stars that we would describe as $\lambda$ Boo stars. We consider a metal-poor star to belong to the $\lambda$ Boo group if we find a depression in the UV. The percentage of metal-weak A stars that show this depression is about 40 to $50 \%$.

Cunha: I have two comments. The first is about the precision to which we might be able to do asteroseismology. Of course the number of modes that we will be able to detect is important, but for A-type stars, I would say that problem of identification of the modes, like Breger was discussing yesterday for the case of $\delta$ Sct stars, and the problem 
for magnetic stars that they are by no means spherically symmetric, will be even more serious obstacles to us than the number of modes that we can detect.

Shibahashi: I don't want to make the problem more complicated. Balona clearly stated that we should have a large number of modes, and we should have clear identifications for these modes, and we should have a good reference model. Mode identification is certainly a very serious problem. If we take into account the difficulty of mode identification, the lack of some eigenfrequencies, and the lack of a good reference model, we cannot say anything definite about the prospects for asteroseismology. What I intended in my talk was to simplify the situation a lot, probably too much. I assumed that the eigenmodes are there, that they are all identified. This is pretty unrealistic, but it seems to me that we simply cannot show the power of asteroseismology if we consider the problems of mode identification, of missing modes, and of poor reference models.

Cunha: Perhaps you misunderstood my comment. What you did was very good; isolating that part is very important. I just want to say that in my opinion that is not what is going to be most in our way for asteroseismology. I was not criticizing what you did.

My other comment was about effective temperature differences in spot models. Going back to the model that I mentioned previously for which we did non-adiabatic calculations, we allowed for differences in effective temperature and luminosity. The differences in effective temperature that we found were rather smaller than you found, because we allowed for luminosity variations as well, and the effect was only a few K. I discussed trying to detect this effect observationally with Petit, who works on cooler stars, and he told me that it would be extremely difficult to detect such a small effect.

ShiBAHASHI: Yes, I think the $500 \mathrm{~K}$ in my estimates may be too much.

Dworetsky: I have a question for Paunzen about the binarity of the $\lambda$ Boo stars. You pointed out that the only A-type companions found so far are also $\lambda$ Boo stars, and no other types of companions are seen. There is probably a good reason for that. If any star that might have been able to become a $\lambda$ Boo star had a solar type companion, the solar wind of that companion would blow away the local ISM and create a protective bubble around both stars, and prevent the accretion of interstellar gas. I think that that might be a reasonable hypothesis to explain that particular feature. Of course, with only 50 or 60 stars known, the statistics are rather slim, but it is surprising that only 10 or $15 \%$ of the stars are binaries. The percentage should be somewhat higher, perhaps around $50 \%$.

PAUnZen: As Corbally told us, if you find a spectroscopic binary with a $\lambda$ Boo component and a cool component, in this system diffusion and mass loss could be responsible for the $\lambda$ Boo star. So we don't insist on the accretion model; in a very old A star in a binary system, the $\lambda$ Boo component might be produced by diffusion and mass loss.

WeIss: I have a question to Shibahashi, and a comment. The question: you based your final analysis on the observability of 200 modes. How do your results scale with the number of observable modes? Perhaps due to the (unknown) excitation mechanism, there simply won't be that many modes, or perhaps, as suggested by some model analyses, we might see even more than 200 modes.

Shibahashi: If I adopt only 100 eigenfrequencies, but with the same range of eigenfrequencies as before, the situation is not so bad. However, if we reduce the range of frequencies substantially, then the result becomes much worse. 
WeIss: And I have a comment to Balona, who raised the question of Q modes for $\lambda$ Boo stars. If I remember the HR diagram that Paunzen showed, the distribution of $\lambda$ Boo stars in the HR diagram is fairly similar to that of the $\delta$ Sct stars, so the curious Q-mode histogram cannot be due to selection effects.

BALOnA: But in the Paunzen diagram, I recall that there are more $\lambda$ Boo stars near the hot end than you would expect. Therefore there is a kind of bias towards the hot end, where the overtones predominate.

PAUnzen: There might be a bias, with about five or six which are going to the hot end. But I don't think that this is really a bias that you see in the Q-values, because if you really look at the distributions of $\delta$ Sct type stars and $\lambda$ Boo stars, you find no $\lambda$ Boo star that could pulsate in the fundamental mode. This might be due to diffusion. It has been suggested by theoretical groups that diffusion does something in the atmosphere that changes the pulsational characteristics of stars, as in Am stars where you hardly find any pulsation. It might be the same effect here. But also you see a shift in the instability strip. This suggests that there may be some related effects due to diffusion in these stars.

KuRTz: First a comment on Paunzen's remarks. I had the same question as Balona about the difference in Q-values. We have seen that there may be a shift in the instability strip from the $\delta$ Sct stars to the $\lambda$ Boo stars. A good way to test the conclusion about the distribution of Q-values would be to take a sample of $\lambda$ Boo stars that overlap the $\delta$ Sct instability strip, balance the numbers, and see if there is still a shift in the Q-values. This would strengthen the result.

The question I have comes from Kochukhov's talk, but it is a question for Bagnulo. Kochukhov found for HR 3831 a magnetic obliquity of 90 degrees. The geometry he found for this star is consistent with the old photometry and the old oblique pulsator model, but it is not consistent with a magnetic model published by Bagnulo which has a very small obliquity. Kochukhov has told me that you have new models of this star which have a larger magnetic obliquity. Can you match Kochukhov's magnetic geometry? Is there consistency in the magnetic fields with what he finds? This is a test of Kochukhov's magnetic angles.

BAGnulo: The answer depends on which geometry you assume. My models depend on the overall morphology that we assume for the magnetic field. If the magnetic field is dominated by a dipolar component, then the dipole axis must be tilted at a very small angle to the rotation axis to fit the observed values of the mean quadratic field. So there are basically two assumptions: that the mean quadratic field modulus measurements are not overestimated, and that the morphology is approximately dipolar. If we assume that there is a strong quadrupolar component, which I think Kochukhov has ruled out, then it might be possible to fit the data with a dipole having a large angle to the rotation axis. But I don't remember at the moment the details of this model. In any case, I think that Kochukhov did suggest that the overall morphology must be roughly dipolar.

KURTz: It would be interesting to see how complex a magnetic morphology you need to shift the dipole obliquity to 90 degrees from the value of 8 degrees that you found. That might need quite a complex magnetic geometry. It would be useful if you could tell us the result.

BAGNulo: I have my laptop here.... 
KocHUKHOV: I would like to comment that there really is a problem of incompatibility between the quadratic field measurements for this star, and other estimates of the field strength. In my Doppler imaging of this star, I estimated the magnetic field strength using magnetic intensification of spectral lines. This was done based on new observational material of high quality, good phase coverage, and polarized radiative transfer calculations. I find that in order to obtain consistent abundances from iron lines, I have to use a magnetic field of the order of $2 \mathrm{kG}$. A field of $11 \mathrm{kG}$ clearly introduces a very large discrepancy into iron abundances derived from different spectral lines. I'm not saying that my results are correct, but I do not think that we should rely on observations of this star done 15 years ago, as Bagnulo did. We should obtain new high-resolution polarization spectra of this star and try to solve this problem.

BREGER: I would like to muddy the waters a little concerning the comparison between the $\lambda$ Boo stars and the $\delta$ Sct stars. The borders of the instability strip are set by the hottest and coolest stars that we know of any type; if we have one single star that is pulsating and that is very hot or very cool, we draw the border beyond that star. The cool border of the $\delta$ Sct instability strip is actually defined by the star $\rho$ Pup, and near it are a huge number of constant stars. Now this situation does not bother us too much, since the temperatures of $\delta$ Sct stars are not that well determined anyway, and $\rho$ Pup has more metals and therefore more line blocking, and so its colour is too red. But in any such comparison this should bother us.

Also, recently the borders of the instability strip have been shifting. They have been shifting to hotter temperatures, as you have for $\lambda$ Boo stars, because we now observe stars with very small amplitudes and very short periods. So I would support Kurtz's suggestion. One should take a definite region in the HR diagram and compare the properties.

KHALACK: I just have a small question about $\gamma$ Equ, about non-LTE effects. Has anyone estimated the non-LTE effects, and would they be strong enough to shift the Pr III and Nd III pulsation depths to the $\mathrm{H}$ line core pulsation depth?

RyABCHIKOVA: In my talk I discussed the non-LTE calculations for Nd II and Nd III. The line depths which were shown by Kochukhov are based on these new non-LTE calculations. The result is that the depth of formation of these Nd III lines is moved closer to the $\mathrm{H} \alpha$ core.

PAUnzen: I have a comment about Breger's comments. If we look at the hot pulsating $\lambda$ Boo stars, we have amplitudes of $0.1 \mathrm{mag}$, which is not observed in hot $\delta$ Sct stars. Even if we shift the instability strip, the characteristics found for the pulsating hot $\lambda$ Boo stars are completely different than those found for the $\delta$ Sct stars. So I guess that there are real differences at the hot border.

TRAMPEDACH: I have a comment on the formation of $\lambda$ Boo stars by accretion from the ISM, and on the bow shock. The key aspect here is what happens to a spectroscopic binary, where the two stars are fairly close to one another. While the system goes through a dense cloud, the stars are also stirring up the ISM, so you would have a completely different picture than just one bullet going through and creating a bow shock. You would have one of the stars going through the slipstream of the other, you would have turbulence occurring. It would be worthwhile to do hydrodynamical calculations on this situation. 
Also you talked about open clusters which have no internal ISM, so there would be no chance of accretion there. Wouldn't some accretion occur as the cluster passes through the galactic plane?

PAUnZEN: Yes, but normally the time scale doesn't work. If an open cluster goes through the galactic plane, the passage is normally much faster than you need for accretion, because the accretion rates are very low. If the accretion rate were high, normally you would detect some signs of this accretion. So the time-scales don't fit.

KUPKA: That ends this discussion. I would like to thank the speakers again. 